\title{
Reverse Transformation of Deformation Induced Martensite in Austenitic Stainless Steel
}

\author{
E. DRYZEK*, AND M. SARNEK \\ Institute of Nuclear Physics, PAS, E. Radzikowskiego 152, 31-342 Kraków, Poland
}

\begin{abstract}
Cumulative isochronal annealing of stainless steel 1.4307 samples deformed by compression was investigated using the Doppler broadening of the annihilation line measurements. Higher level of plastic deformation is accompanied not only by higher concentration of defects but also by higher amount of $\alpha^{\prime}$ martensite. The annealing studies revealed creation of defects in the temperature range between $450{ }^{\circ} \mathrm{C}$ and $650{ }^{\circ} \mathrm{C}$ pointing out the reverse transition of $\alpha^{\prime}$ martensite to austenite. This is confirmed by magnetic susceptibility and microhardness measurements.
\end{abstract}

DOI: $10.12693 /$ APhysPolA.125.710

PACS: 78.70.Bj, 81.05.Bx

\section{Introduction}

Austenitic stainless steels (SSs) are widely used in engineering applications due to their mechanical properties, weldability and excellent corrosion resistance. Therefore controlling their microstructure evolution, and physical and mechanical properties is very important. Austenitic SSs are paramagnetic in the annealed, fully austenitic condition. Plastic deformation of austenitic SS, apart from introducing crystal defects, can induce also transformation from austenite to martensite [1] depending on the steel composition. Two types of deformation-induced martensite can form: $\alpha^{\prime}$ (bcc) which is ferromagnetic and $\varepsilon$ (hcp) which is paramagnetic. Then changes in magnetic properties due to the formation of $\alpha^{\prime}$ martensite are frequently used for its detection [2]. There was found that for low deformation levels the amount of $\varepsilon$-martensite is predominant in proportion to $\alpha^{\prime}$-martensite [3]. When deformation level increases, the quantity of $\alpha^{\prime}$-martensite grows. The transformation sequence has been proposed to be $\gamma \rightarrow \varepsilon \rightarrow \alpha^{\prime}$ [1]. The formation of deformation-induced $\alpha^{\prime}$ martensite significantly affects the mechanical behavior of austenitic SS by enhancing work hardening. Deformation induced martensitic transformation is extensively studied because of its practical applications. It is also important to understand the mechanism by which this phase transformation occurs. Reverse transformation of deformation induced martensite $\alpha^{\prime} \rightarrow \gamma$ in the austenitic SS is less studied than its formation [4] and its mechanism has not yet been clarified. The $\alpha^{\prime} \rightarrow \gamma$ was reported as resulting in formation of austenite with high dislocation density [5] or in micro-twins and stacking faults [6]. There was demonstrated the formation of ultra-fine austenite grains [7] in metastable austenitic SS which can be used for grain refinement $[8,9]$. It opens up the possibility of using high strength austenitic SS in structural applications.

\footnotetext{
*corresponding author; e-mail: ewa.dryzek@ifj.edu.pl
}

The positron annihilation techniques are well-known experimental methods used for studies of defects in crystalline matter. Positrons implanted into a metallic system localize in regions where electron density is lower than in a perfect crystal lattice, i.e. open volume defects, and then annihilate with electrons. The annihilation radiation delivers information on the local electron density and the electron momentum distribution in the sites where the annihilation takes place. Crystal lattice defects generated during plastic deformation, i.e. open-volume defects such as vacancies and their agglomerates and to some extent dislocations can be identified and investigated using positron annihilation techniques. They have been used for example for detection of the subsurface zone in the stainless steel created by sliding or different methods of cutting $[10,11]$. The defects can be created also during phase transitions and those can be also detected by positron annihilation methods.

The aim of the presented investigations is to study annealing behavior of deformed SS 1.4307 (EN) in terms of annealing of defects detected by positron annihilation methods paying close attention to the influence of reverse transformation of deformation induced martensite on the obtained dependences. The studies are complemented by microhardness and magnetic susceptibility measurements.

\section{Experimental details}

The material under investigation was a commercial SS 1.4307 (EN) equivalent to AISI 304L. Table shows the chemical composition of the samples determined using an atomic emission spectrometer with spark excitation. Samples in shape of discs, $4 \mathrm{~mm}$ high and $10 \mathrm{~mm}$ in diameter, were annealed for $1 \mathrm{~h}$ in the flow of $\mathrm{N}_{2}$ gas at a temperature of $700^{\circ} \mathrm{C}$, and then slowly cooled to room temperature to remove any deformation effects due to cutting and surface polishing. After annealing, the surface layer was removed by etching in hydrofluoric acid and $25 \%$ solution of nitric acid in distilled water. The samples were plastically deformed using hydraulic press getting thick- 
ness reduction equal to $16 \%$. Then the isochronal cumulative annealing for $1 \mathrm{~h}$ in the temperature range from room temperature to $725^{\circ} \mathrm{C}$ was performed. The X-ray diffraction measurements were carried out in a Philips X-Pert diffractometer using $\mathrm{Cu} K_{\alpha}$ radiation. After each annealing, the positron measurement was performed using a Doppler broadening (DB) spectrometer with a coaxial HPGe (high purity germanium) detector with interpolated energy resolution of $1.4 \mathrm{keV}$ full width at half maximum (FWHM) at $511 \mathrm{keV}$. For DB measurements the ${ }^{68} \mathrm{Ge} /{ }^{68} \mathrm{Ga}$ positron source was used.

TABLE

The chemical composition of the studied samples (wt\%).

\begin{tabular}{c|c|c|c|c|c|c|c|c|c|c}
\hline \hline Steel & $\mathrm{C}$ & $\mathrm{Si}$ & $\mathrm{Mn}$ & $\mathrm{P}$ & $\mathrm{S}$ & $\mathrm{Cr}$ & $\mathrm{Ni}$ & $\mathrm{Mo}$ & $\mathrm{Cu}$ & $\mathrm{V}$ \\
\hline 1.4307 & 0.012 & 0.29 & 1.13 & 0.028 & 0.026 & 18.37 & 8.12 & 0.36 & 0.35 & 0.09
\end{tabular}

There were performed also the measurements of magnetic properties of the deformed sample using the susceptometer described in Ref. [12]. It allowed us to determine relative changes of the susceptibility. The maximum of magnetic induction used in the measurement was equal to $20 \mathrm{mT}$. The measurements of Vickers microhardness were carried out using Zeiss (Neophot 30) microscope at the load of $100 \mathrm{~g}$. There were performed additional positron lifetime measurements for the initially annealed sample, the deformed sample and the sample after annealing in temperature range of martensite reversion. The positron lifetime spectra of more than $1.5 \times 10^{6}$ counts were measured using a fast-fast spectrometer with $\mathrm{BaF}_{2}$ scintillators and ${ }^{22} \mathrm{Na}$ isotope source enveloped in a $7 \mu \mathrm{m}$ thick kapton foil. The time resolution of the system was $280 \mathrm{ps}$ (FWHM). All the obtained spectra were analyzed using the LT code [13].

\section{Results and discussion}

The positron lifetime measured for the initially annealed reference sample was equal to $(109 \pm 1) \mathrm{ps}$, which may be attributed to annihilation of positrons in the perfect lattice. The mean positron lifetime for the sample after plastic deformation was equal to $166 \pm 1 \mathrm{ps}$, which is lower than the lifetime of positron trapped in the vacancy in the perfect lattice of iron, i.e. 175 ps [14]. This may suggest that the vacancies in which trapped positrons are localized in the vicinity of dislocation lines, where atomic surrounding is distorted. This gives lower values of positron lifetime $[15,16]$.

Figure 1 presents the results of DB measurements for isochronal annealing of the steel sample deformed to thickness reduction $16 \%$. For comparison, there are also shown the results obtained for the sample deformed to thickness reduction $6 \%$ [17]. In Fig. 1a there is depicted the dependence of the $S$-parameter, which is defined as the ratio of the area under the fixed central part of the annihilation line to the area under the whole annihilation line, on annealing temperature. The $S$-parameter is sensitive to the annihilation of positrons with low momentum electrons which are present in open volume defects.
For the sample with higher thickness reduction the initial value of the $S$-parameter is higher, which indicates higher defect concentration. For the sample with lower thickness reduction, i.e. $6 \%$, an increase of the $S$-parameter for annealing temperatures between $100^{\circ} \mathrm{C}$ and $200^{\circ} \mathrm{C}$ can be noticed. This increase in $S$-parameter may be caused by additional defects created by $\varepsilon$-martensite reversion [18]. Although $\varepsilon$-phase was not revealed in diffractions patterns presented in Fig. 2, our later measurement for the sample with similar thickness reduction performed specifically in order to search for $\varepsilon$-martensite in the deformed sample confirmed its presence.

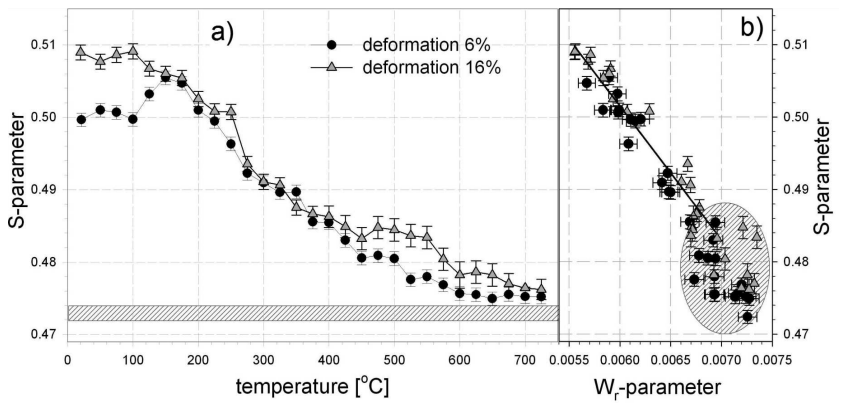

Fig. 1. (a) The $S$-parameter as a function of annealing temperature for $1 \mathrm{~h}$ isochronal annealing of 1.4307 (EN) stainless steel deformed to thickness reduction $16 \%$. For comparison there is shown also the dependence for the sample deformed to thickness reduction $6 \%$. The hatched area shows the value of the $S$-parameter for the initial sample before deformation. In (b), the $S$-parameter as a function of the $W_{r}$-parameter. The solid straight line was fitted to the experimental points for temperature range from room temperature to $400^{\circ} \mathrm{C}$. The hatched oval area indicates the scattered data points for higher temperatures.

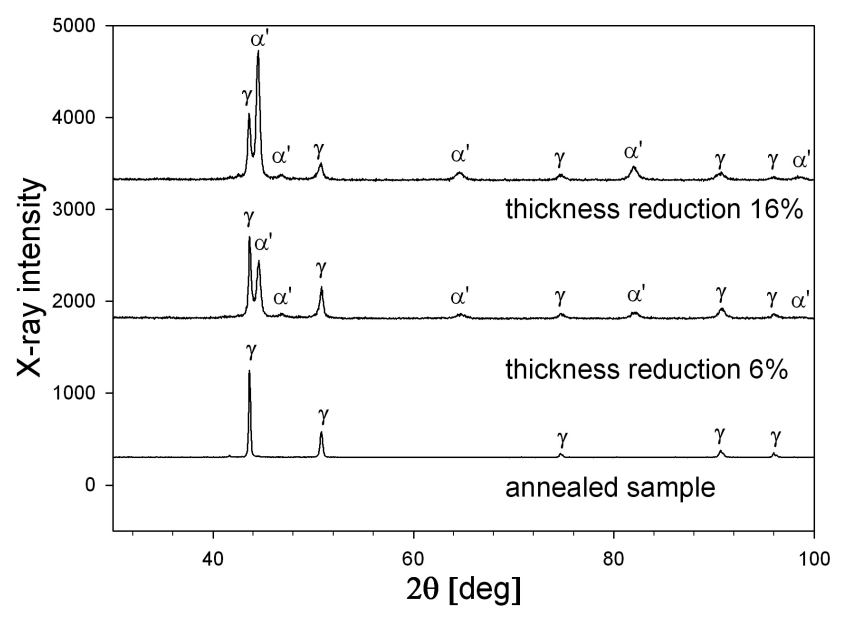

Fig. 2. X-ray diffraction patterns for the initial annealed sample before deformation and the samples deformed to thickness reduction $6 \%$ and $16 \%$.

Decrease of the $S$-parameter with temperature for temperatures above $200{ }^{\circ} \mathrm{C}$ indicates annealing of de- 
fects induced by plastic deformation which may be described for example by vacancy migration model [19] in which annealing of the deformed sample causes diffusion of vacancies detected by positrons to grain boundaries or other sinks where they annihilate. In our previous paper, there was shown that the vacancy migration model does not describe well the dependence obtained for the sample deformed to thickness reduction $6 \%$ [17], although this model was successfully applied for other steels [20,21]. This may be caused by the fact that the obtained $S$-parameter temperature dependence is influenced by two processes, i.e. the migration of vacancies to grain boundaries and sinking there and the $\alpha^{\prime}$ martensite reversion to austenite which can generate additional defects.

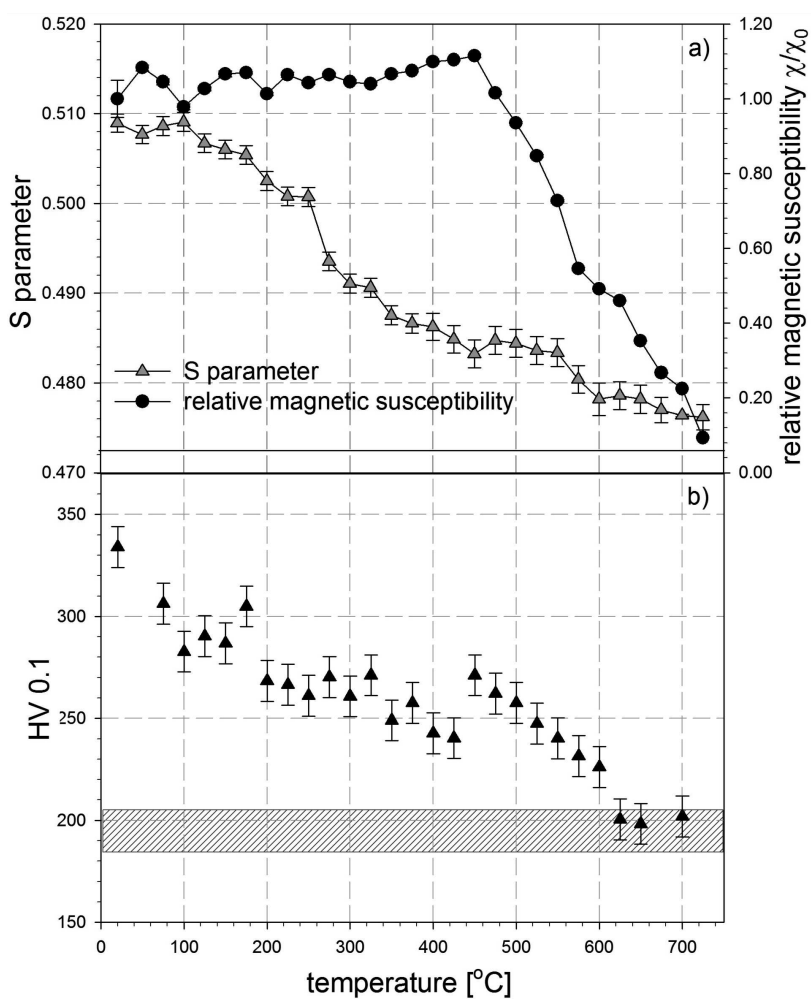

Fig. 3. (a) The annealing temperature dependence of the relative magnetic susceptibility $\chi / \chi_{0}$, (right axis) and the $S$-parameter as a function of annealing temperature, (left axis) for the sample deformed to thickness reduction $16 \%$. The horizontal solid line indicates the $\chi / \chi_{0}$ value for the annealed sample. In (b) Vickers microhardness as a function of annihilation temperature. The hatched area shows the value of the microhardness for the initial sample before deformation.

Higher level of plastic deformation, i.e. the higher thickness reduction, is accompanied not only by higher concentration of defects but also by higher amount of $\alpha^{\prime}$ martensite. For the sample deformed to higher thickness reduction, i.e. $16 \%$, the values of the $S$-parameter for the temperature range between $450^{\circ} \mathrm{C}$ and $675^{\circ} \mathrm{C}$ constitute a hump in comparison to the dependence for the sample with lower thickness reduction, i.e. $6 \%$. Reverse transformation of deformation induced $\alpha^{\prime}$ martensite to austenite in this temperature range is confirmed by the starting at $450^{\circ} \mathrm{C}$ decrease of the relative magnetic susceptibility $\chi / \chi_{0}$, where $\chi_{0}$ is the magnetic susceptibility of the deformed sample before the annealing procedure (Fig. 3a). The microhardness measured for this sample as a function of annealing temperature decreases in whole measurement temperature range up to $625^{\circ} \mathrm{C}$ (Fig. $3 \mathrm{~b}$ ). However, this decrease becomes steeper above $450^{\circ} \mathrm{C}$, which coincidences with the start of the susceptibility decrease. For temperature above $600{ }^{\circ} \mathrm{C}$ it reaches the value measured for the annealed sample.

The creation of new defects in the region of reversed transition of $\alpha^{\prime}$ martensite can be noticed also in the $W_{r}-S$ dependence which is shown in Fig. 1b. The $W_{r}$ parameter is defined as the ratio of the area under the fixed wing region of the annihilation line to the area under the whole annihilation line. This parameter reflects the annihilation of positrons with high momentum electrons. The straight line fitted to the $W_{r}-S$ dependence suggests that the type of positron trapping defects does not change but their concentration changes. The straight line was fitted to the data obtained for both samples in the temperature range between room temperature and $400{ }^{\circ} \mathrm{C}$. For higher annealing temperatures the slope of the dependence changes, but the experimental points are scattered. The change of the slope may be interpreted as a change of the main type of defects localizing positrons. The mean positron lifetime for the sample after annealing at $450{ }^{\circ} \mathrm{C}$ was equal to $(129 \pm 1)$ ps and it is higher than the positron lifetime at edge dislocations in $\alpha$ iron equal to 117 ps [16].

\section{Conclusions}

The plastic deformation of the SS sample induces crystal lattice defects but also the transformation to the $\alpha^{\prime}$ martensite. The isochronal annealing of the plastic deformed SS samples was investigated by the measurements of the annihilation line shape parameter, i.e., $S$-parameter. The dependence shows the gradual annealing of the defects induced by deformation, but in the temperature range between 450 and $650^{\circ} \mathrm{C}$ the process of new defect generation is noticed. It can be attributed to $\alpha^{\prime}$ martensite reversion. This process was confirmed by magnetic susceptibility and Vickers microhardness measurements. Both quantities gradually decrease in this temperature range.

\section{References}

[1] P.L. Mangonon, G. Thomas, Metall. Trans. 1, 1577 (1970).

[2] S.S.M. Tavares, M.R. da Silva, J.M. Neto, S. Miraglia, D. Fruchart, J. Magn. Magn. Mater. 242-245, 1391 (2002).

[3] V. Seetharaman, R. Krishnan, J. Mater. Sci. 16, 523 (1981). 
[4] F. Haeßner, R.L. Plaut, A.F. Padilha, ISIJ Int. 43, 1472 (2003).

[5] H. Smith, D.R.F. West, J. Mater. Sci. 8, 1413 (1973).

[6] H. Kessler, W. Pitsch, Acta Metall. 15, 401 (1967).

[7] S. Rajasekhara, L.P. Karjalainen, A. Kyröläinen, P.J. Ferreira, Mater. Sci. Eng. A 527, 1986 (2010).

[8] A. Di Schino, I. Salvatori, J.M. Kenny, J. Mater. Sci. 37, 4561 (2002).

[9] K.H. Lo, C.H. Shek, J.K.L. Lai, Mater. Sci. Eng. $R$ 65, 39 (2009).

[10] J. Dryzek, P. Horodek, M. Wróbel, Wear 294-295, 264 (2012).

[11] P. Horodek, J. Dryzek, M. Wróbel, Tribol. Lett. 45, 341 (2012).

[12] R. Davis, M. Gläser, Metrologia 40, 339 (2003).

[13] J. Kansy, Nucl. Instrum. Methods Phys. Res. A 374, 235 (1996).
[14] A. Vehanen, P. Hautojärvi, J. Johansson, J. YliKauppila, Phys. Rev. B 25, 762 (1982).

[15] K. Hori, K. Koike, R. Oshima, Appl. Surf. Sci. 242, 304 (2005).

[16] Y. Kamimura, T. Tsutsumi, K. Kuramoto, J. Phys. Soc. Jpn. 66, 3090 (1997).

[17] E. Dryzek, M. Sarnek, K. Siemek, Nukleonika 58, 215 (2013).

[18] A.F. Padilha, R.L. Plaut, P.R. Rios, ISIJ Int. 43, 135 (2003).

[19] J. Dryzek, Mater. Sci. Forum 255-257, 533 (1997).

[20] J. Dryzek, C. Wesseling, E. Dryzek, B. Cleff, Mater. Lett. 21, 209 (1994).

[21] U. Holzwarth, A. Barbieri, S. Hansen-Ilzhöfer, P. Schaaff, M. Haaks, Appl. Phys. A 73, 467 (2001). 